\title{
Norois
}

Environnement, aménagement, société

248 | 2018

Observer l'impact des lignes à grande vitesse : acteurs et territoires du grand Ouest français

\section{L'impact de la LGV Bretagne-Pays de la Loire sur le développement régional : une évaluation prospective à l'horizon 2040}

The Impact of Bretagne-Pays de la Loire HSL on Regional Development: A

Prospective Assessment for 2040

Guy Baudelle, Ingrid Brugioni et Arnaud Lepetit

\section{(2) OpenEdition}

Journals

Édition électronique

URL : https://journals.openedition.org/norois/6842

DOI : 10.4000/norois.6842

ISSN : $1760-8546$

Éditeur

Presses universitaires de Rennes

Édition imprimée

Date de publication : 5 novembre 2018

Pagination : 15-36

ISBN : 978-2-7535-7627-8

ISSN : 0029-182X

Référence électronique

Guy Baudelle, Ingrid Brugioni et Arnaud Lepetit, «L'impact de la LGV Bretagne-Pays de la Loire sur le développement régional : une évaluation prospective à l'horizon 2040 », Norois [En ligne], 248 | 2018, mis en ligne le 05 novembre 2020, consulté le 14 janvier 2022. URL : http://journals.openedition.org/ norois/6842 ; DOI : https://doi.org/10.4000/norois.6842 


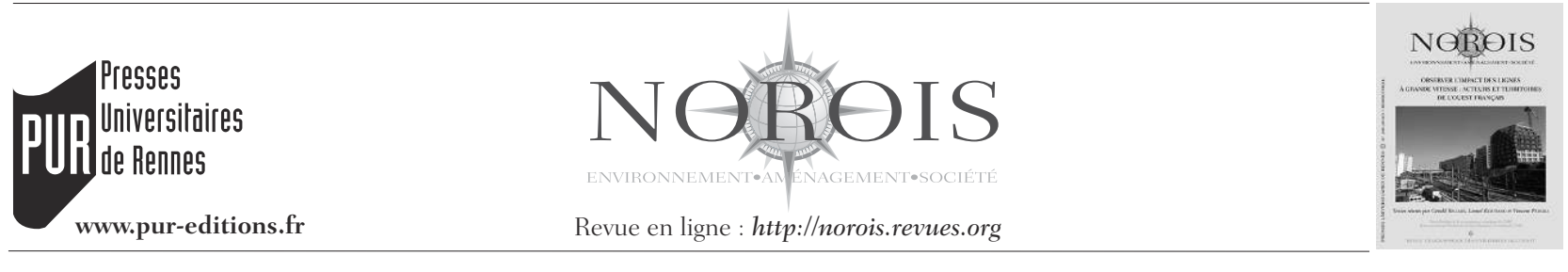

\title{
L'impact de la LGV Bretagne-Pays de la Loire sur le développement régional : une évaluation prospective à l'horizon 2040
}

\author{
The Impact of Brittany-Pays de la Loire HSL on Regional Development: \\ A Prospective Assessment for 2040
}

Guy Baudelle ${ }^{* a}$, Ingrid Brugioni ${ }^{\text {b }}$ et Arnaud Lepetit ${ }^{\text {a }}$

\footnotetext{
* Auteur correspondant : tél. : 0950245469

a ESO-Rennes, UMR 6590 ESO, UFR Sciences Sociales, Université Rennes - 2, place du Recteur-Henri-Le-Moal, CS 24307,35043 Rennes Cedex, France.(guy.baudelle@univ-rennes2.fr, arnaud.lepetit@univ-rennes2.fr)

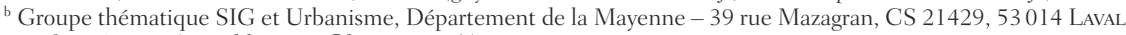

Cedex, France. (ingrid.brugioni@lamayenne.fr)
}

Résumé : Une revue succincte de la littérature géographique et économique montre que l'état des connaissances actuelles n'autorise pas de conclusions définitives quant à l'effet des infrastructures de transport - et en particulier de la Grande vitesse ferroviaire - sur le développement régional. Cette incertitude scientifique confère à la prospective territoriale un intérêt stratégique. C'est pourquoi on présente la méthode et les résultats d'une prospective des enjeux d'aménagement que soulèvent la ligne à grande vitesse BretagnePays de la Loire et les projets associés pour l'Ouest de la France à partir de cinq scénarios à l'horizon 2040.

Abstract: A brief review of the geographical and economic literature indicates that the current state of knowledge does not allow definitive conclusions about the effect of transport infrastructure - particularly high-speed rail - on regional development. Given this scientific uncertainty, territorial foresight is of strategic interest. This is why the method and results of a foresight study of planning stakes raised in Western France by the Brittany-Pays de la Loire High-speed line and related projects are presented from five scenarios by 2040.

Mots clés : train à grande vitesse - transport collectif - développement régional - prospective - scénarios

Keywords: high speed train - public transport - regional development - foresight - scenarios 


\section{INTRODUCTION}

La Ligne à grande vitesse Bretagne Pays-de-laLoire (LGV BPL) mise en service en juillet 2017 prolonge sur $182 \mathrm{~km}$ l'axe antérieur, de l'est du Mans jusqu’à Rennes. La «virgule de Sablé » la complète en assurant la connexion en direction de Nantes entre Le Mans et Laval (figure 1 - planche I).

Cette infrastructure majeure est complétée par des travaux dits « de relèvement de vitesse » sur les lignes vers Brest et Quimper à l'ouest de Rennes, la modernisation d'autres axes et l'aménagement de pôles d'échanges multimodaux dans le cadre du projet régional dit « Bretagne Grande Vitesse ${ }^{1}$ ».

A cela s'ajoute le projet global d'amélioration de la desserte régionale dit Liaisons nouvelles Ouest Bretagne-Pays de la Loire (LNO BPL) qui vise à amplifier les effets de la LGV en structurant le réseau ferroviaire à long terme (2030 et au-delà). La procédure de débat public close début 2015 a validé les cinq objectifs de la $\mathrm{SNCF}^{2}$.

Les espoirs de retombées territoriales de la LGV proprement dite sont à la mesure de l'importance de l'investissement (3,3 milliards $€$ ) qui justifie la mise en place d'un Observatoire socio-économique pour tirer le bilan des résultats économiques et sociaux (Bertrand et Billard, 2018). Si l'évaluation de l'impact environnemental des grandes infrastructures est assez rodée, l'appréciation de l'effet économique est paradoxalement moins éprouvée sur un plan théorique.

Le panorama de la littérature consacrée aux effets des grands équipements - et plus spécifiquement des Lignes à grande vitesse - sur la croissance régionale et urbaine que nous proposons révèle en effet un cruel manque de certitudes malgré l'ampleur des publications en géographie et économie spatiale : " the conclusion [...] is essentially no conclusion» résume, fataliste, Vickerman (2017). C'est pourquoi on souligne ensuite l'intérêt de la prospective territoriale : si l'état des connaissances n'autorise pas de conclusions assurées, il convient d'affronter les défis correspondants en s'attachant à la construction de scénarios différenciés conçus comme des outils d'anticipation. Il ne s'agit pas de prévoir l'avenir mais d'identifier les conséquences possibles des mégaprojets sur le devenir des territoires. On présente

1. Cf. [http://transports.bretagne.bzh/bretagne-grande-vitesse/] et la carte correspondante : [http://transports.bretagne.bzh/wp-content/ uploads/2016/03/CarteBGV-1024x554.png].

2. Cf. [http://www.lnobpl.fr/objectifs]. la méthode, les résultats et les implications pour l'aménagement d'un travail de prospective sur les enjeux liés au projet LNO BPL à l'horizon 2040 réalisé pour Réseau Ferré de France (RFF) (désormais SNCF Réseau) (Baudelle et al., 2014). L’article rappelle l'intérêt de ce type d'exercice pour l'action publique, expose la méthode adoptée et présente les principaux scénarios élaborés pour les deux régions concernées.

\section{LES EFFETS INCERTAINS DE LA GRANDE VITESSE FERROVIAIRE}

\section{Pas d'impact avéré sur la croissance régionale et urbaine}

Les gains de temps absolus résultant du projet LGV BPL (et des premiers travaux de BGV associés) sont plus importants pour les villes les plus éloignées de la capitale mais le raccourcissement relatif (en pourcentage) s'avère plus net pour Rennes et Laval (tableau 1) (Nègre, 2018).

Les acteurs des territoires desservis attendent logiquement des effets positifs des rapprochements physiques induits par la LGV (Agences..., 2010; Salmon, 2013; AUDIAR, 2016 ; Nègre, 2018). En termes théoriques, la contraction de l'espace-temps transforme en effet le système de villes régional (Brotchie, 1991; Sasaki et al., 1997; Bretagnolle et al., 1998; Rozenblat, 2004; Bretagnolle, 2003 et 2009; Berroir et al., 2012; Levinson, 2012). La relation entre accessibilité et croissance urbaine et régionale est en outre reconnue (Rietveld et Bruinsma, 1998; Banister et Berechman, 2001 ; Duranton et Turner, 2012; Levinson, 2012) tout comme l'impact d'une desserte ferroviaire (ou de son absence) sur la croissance différentielle des villes en longue durée (Auphan, 1975; Bellet et al., 2012).

Ces assurances apparentes devraient inciter à la prudence dans le cas de la LGV BPL, pour deux motifs. Le premier est le temps gagné. On considère en effet généralement que l'impact est proportionnel au temps gagné (à défaut de linéarité parfaite). Or, les taux d'accélération des liaisons avec la capitale sont modestes (tableau 1, colonne de droite). Celui de Rennes (réduction de près d'un tiers du temps de trajet) est analogue au taux du TGV Atlantique de 1989 mais bien inférieur à celui de la LGV Nord 


\begin{tabular}{|c|c|c|c|c|}
\hline Destination & Avant LGV & Après LGV & Gain $(\mathbf{m n})$ & Gain (\%) \\
\hline Rennes & $2 \mathrm{~h} 04$ & $1 \mathrm{~h} 25$ & 39 & 31 \\
\hline Laval & $1 \mathrm{~h} 32$ & $1 \mathrm{~h} 1 \mathrm{l}$ & 21 & 23 \\
\hline Angers & $1 \mathrm{~h} 30$ & $1 \mathrm{~h} 24$ & 6 & 7 \\
\hline Nantes & $2 \mathrm{~h} 05$ & $1 \mathrm{~h} 56$ & 46 & 18 \\
\hline Brest & $4 \mathrm{~h} 11$ & $3 \mathrm{~h} 25$ & 45 & 18 \\
\hline Quimper & $4 \mathrm{~h} 16$ & $3 \mathrm{~h} 31$ & 9 & 7 \\
\hline
\end{tabular}

Tableau 1 : Quelques gains de temps avec Paris consécutifs à la Ligne à grande vitesse BPL (trajets les plus rapides mi-2017) (Source : SNCF)

Some time savings to Paris following the Bretagne-Pays de la Loire High Speed Line (fastest journeys in mid-2017) (Source: SNCF)

pour Lille $(50 \%)$. Ce moindre gain relatif pourrait cependant être compensé par un effet de réseau plus marqué par rapport aux années 1980 dans la mesure où la LGV de 2017 donne accès à un nombre plus élevé de destinations et à un système LGV plus vaste, ce qui devrait en démultiplier les effets. Le second motif de prudence est le caractère tardif de l'ouverture de la ligne, sachant que la date de réalisation d'une infrastructure est discriminante, les villes précocement desservies étant susceptibles de creuser l'écart avec les autres. Or, d'autres villes ont été intégralement reliées à Paris par LGV bien plus tôt (Lyon dès 1983, Lille en 1993, Marseille en 2001). L'effet espéré de la LGV pourrait donc se révéler moindre.

La plupart des élus ont traditionnellement des attentes excessives par rapport aux nouvelles infrastructures alors que la recherche met en garde contre toute «LGVmania » (Crozet, 2015). La question de l'impact territorial des grands projets de transport terrestre n'est pourtant pas nouvelle, elle a émergé dès les premières liaisons navigables et ferrées. Les premières LGV ont suscité les mêmes interrogations (Offner, 1993). Des chercheurs questionnent même rétrospectivement les bénéfices sociaux de la Via Appia (Berechman, 2003).

L'appréciation diffère selon les disciplines, les méthodologies, les familles de modèles, les terrains investis et les échelles d'analyse spatiale et temporelle (Oosterhaven et Knaap, 2003; Jensen-Butler et Madsen, 2005). L'évaluation d'un projet analogue à la LGV BPL entre Schiphol et Groningue varie ainsi fortement avec le type de modélisation (Oosterhaven et Knaap, 2003; Elhorst et al., 2004; Vickerman, 2006). La plupart des études observent certes l'effet positif des LGV mais divergent sur son ampleur et sa portée (Vickerman, 1997 et 2006; Gutiérrez, 2001 ; Levinson, 2012 ; Loukaitou-Sideris et al., 2013; Chen et Abreu e Silva, 2014). La géographie théorique a ainsi nuancé sinon invalidé la relation apparemment si bien établie entre (qualité de la) desserte et développement urbain (Pumain, 1982 ; Bretagnolle, 1999 et 2009). De même, les fameux effets structurants des LGV dénoncés de longue date (Offner, 1993 et 2009) ont été qualifiés de «mythologies politico-journalistiques » (Offner, 2014), signe d'une absence de certitude scientifique sur les effets des grands équipements de transport (CEMT, 2001 ; Bazin et al., 2006 ; Vickerman, 2006; Bruinsma et al., 2008; Ureña et al., 2009; Wang et Charles, 2010; SETEC, 2010; Rus, 2012; Mignerey, 2012; Delaplace et Dobruszkes, 2013; Baudelle, 2016; Vickerman, 2017; Button, 2017; Nègre, 2018).

Ainsi, si le transport est «essentiellement [...] une demande résultant de l'activité économique » (JensenButler et Madsen, 2005), l'effet inverse supposé du transport sur la croissance régionale s'en trouve minimisé. Les modèles s'efforçant d'évaluer les effets des LGV sur l'économie régionale peinent à modéliser l'ensemble du système d'offre et de demande (Jensen-Butler et Madsen, 2005). Le principal obstacle, classique, tient à la difficulté de faire la part entre l'incidence directe de l'amélioration du transport et celles imputables au contexte économique général (Crozet, 2015). Les modalités habituelles de l'évaluation coût-bénéfice s'en trouvent remises en cause (CEMT, 2002 ; Berechman, 2009; Crozet, 2014 ; Delaplace et Dobruszkes, 2016; Vickerman, 2017), expliquant le scepticisme sur l'impact de la grande vitesse ferroviaire (Vickerman et al., 1999). Ces calculs se limitent en outre aux effets sur le 
marché du transport et ne renseignent pas sur l'impact territorial. La sophistication croissante des modèles est loin d'avoir éliminé toute controverse, vu l'ambiguïté persistante de certains résultats (effet positif sur l'emploi mais négatif pour le PIB) (Chen et Abreu e Silva, 2013), au point que mettre en évidence des relations de cause à effet serait impossible (Frémont in Offner, 2014) ou du moins difficile à démontrer vu la complexité des mécanismes de rétroaction (Jensen-Butler et Madsen, 2005), mal prise en compte par les approches traditionnelles en termes d'externalités (Rietveld et Bruinsma, 1998; Banister et Berechman, 2001). On constate bien une corrélation entre progrès du trafic et expansion économique mais on ignore toujours lequel engendre l'autre. L'introduction de SIG dans la simulation de scénarios régionaux d'impact à partir de modèles de potentiel ne résout pas les interrogations de base relatives à l'estimation de l'intensité et de la portée des interactions spatiales dans la mesure des effets de débordement (Gutiérrez et al., 2010). La Nouvelle économie géographique n'a pas plus de certitudes quant aux effets de l'amélioration de l'accessibilité sur les performances régionales, très dépendants de la situation initiale (Fujita et al., 1999; Cheng et al., 2013; Vickerman, 2017). Au terme d'une revue des méthodes, un spécialiste estime les bénéfices économiques probables mais très variables et difficiles à prévoir (Vickerman, 2006). De remarquables échanges entre chercheurs montrent les progrès mais aussi les insuffisances persistantes des méthodes, laissant un « goût amer » aux décideurs (OCDE, 2008).

Dans ces conditions, on ne saurait s'étonner si des projets de LGV soulèvent d'aussi fortes polémiques dans de nombreux pays (Amtrak, 2012; Delaplace et Dobruszkes, 2016 ; Nègre et Baudelle, 2016; Button, 2017). En Espagne, les déboires de la grande vitesse ont conduit à de nombreux tests contradictoires (Esteban 1998; Gutiérrez, 2001 ; Gutiérrez Puebla, 2004; Bellet et al., 2010 ; Gutiérrez et al., 2010; Terrin, 2011; Garmendia et al., 2012a; Facchinetti-Mannone et al., 2013; Richer, 2014). Leur caractère récurrent trahit des savoirs mal assurés. L'évaluation de l'impact régional des LGV doit au final se méfier « de la rhétorique du développement régional » car une LGV n'est pas en soi bonne ou mauvaise, il faut en évaluer l'utilité sociale au cas par cas (Rus, 2012).
Un certain nombre d'enseignements pour le développement territorial peuvent malgré tout être tirés (Huart, 1994; SETEC, 2010 ; Mignerey, 2012; Quéré, 2013; Nègre et Baudelle, 2016). Une LGV n'a pas d'effet isolément et doit s'accompagner d'autres mesures (opérations d'urbanisme, programmes de bureaux, amélioration des transports régionaux) (Van den Berg et Pol, 1998; Bazin et al., 2006; Bellet et al., 2012; Meer et al., 2012). Mais si elle amplifie souvent les potentialités des territoires desservis, elle occasionne rarement une inversion de tendance (SETEC, 2010) comme en témoignent les aléas de quartiers d'affaires sortis dans une conjoncture défavorable que la LGV n'a pu suffire à compenser (Ménerault et Barré, 2001 ; Plassard, 2003; Bazin et al., 2011 ; Delaplace et al., 2013; Bellet Sanfeliu et Santos Ganges, 2016; Nègre et Baudelle, 2016; Baudelle, 2016). L'urbaniste rennais Christian Lepetit estime même dans la presse que le TGV s'est tellement banalisé qu'il n'a plus d'effet d'entraînement, ce qui le rend «indispensable mais indifférenciant ». L'état de l'art n'autorise donc pas de conclusions arrêtées sur la dynamisation des territoires par une LGV.

\section{Les spécificités du contexte régional}

On ne saurait de ce fait négliger les spécificités du contexte géographique et temporel : la construction d'une LGV entre de petites localités (Vitré et Sablé, 15000 habitants), une ville moyenne (Laval) et une grande agglomération (Rennes) situées entre 200 et $350 \mathrm{~km}$ à l'ouest de Paris à la fin des années 2010 . Cette situation est particulière car les principales LGV concernent des régions plus fréquentées et des pôles plus importants (Lyon, Lille, Marseille). Les LGV ayant vocation à relier des métropoles, la littérature scientifique les a logiquement privilégiées, la recherche sur les autres strates urbaines étant moins abondante sauf pour les gares périphériques (Facchinetti-Mannone, 2009; Bazin et al., 2013; Richer, 2014; Mohino et al., 2014). Les études sur le TGV Atlantique ont plus de 20 ans et portaient sur une seule ville (Chevalier, 1995-1997; Dornbusch, 1997). Des régions sous-métropolisées ont certes été étudiées en Espagne mais la densité y est bien plus faible (Esteban, 1998; Gutiérrez, 2001 ; Bellet et al., 2010; Gutiérrez et al., 2010; Garmendia et al., 2012a). Les liaisons des années 2000 et 2010 ont 
pour leur part fait l'objet d'investigations difficilement transposables car parcellaires (immobilier, tourisme) et pour des axes comportant plusieurs gares extra-urbaines (Bazin et al., 2004, 2010 et 2013; Saladié et al., 2016). L'enjeu est de taille car le bilan LOTI du TGV Atlantique en avait pointé l'impact limité sur la dynamique urbaine (Chapulut, 2001), y compris à Nantes (Dornbusch, 1997).

Les travaux conduits dans le cadre de l'Observatoire contribuent d'autant mieux à combler cette lacune que s'exprime la crainte récurrente d'un phénomène de pompe aspirante par Paris (Klein, 1998). La LGV avantagerait les grands centres au détriment des périphéries traversées (Rus et Nash, 2007), engendrant métropolisation (Alabalate et Bel, 2010; Garmendia et al., 2012a) et recentralisation (Bél i Queralt, 2010). Mais comme ce mouvement centripète est contrebalancé par la force centrifuge de moindres coûts salariaux, immobiliers et fonciers en province, les avis divergent selon les auteurs (Puga, 2002; Hall, 2009; Garmendia et al., 2012b; Stein, 2013; Loukaitou-Sideris et al., 2013; Vickerman, 2014; Ahlfeldt et Feddersen, 2015; Baudelle, 2016; Button, 2017 ; Nègre, 2018).

\section{UNE PROSPECTIVE TERRITORIALE À 2040 : MOTIVATION ET MÉTHODOLOGIE}

\section{De l'utilité stratégique de la prospective territoriale}

La recherche sur l'impact des LGV sur la croissance régionale et urbaine ne livre donc pas de réponse univoque sur leurs éventuels « effets structurants ", directs ou non (Vickerman, 2006), en dépit d'une stylisation parfois poussée et d'efforts théoriques et empiriques. Cette incertitude peut surprendre au regard du niveau élevé de dépenses et d'espoirs en termes de développement territorial. Elle est pénalisante car investisseurs et élus ont besoin d'anticiper vu la forte inertie des infrastructures lourdes du fait de l'ampleur des chantiers et de la longueur des procédures. Les acteurs territoriaux se trouvent donc confrontés à un avenir rendu encore plus incertain par l'instabilité technologique des systèmes de transport.

C'est pourquoi RFF a souhaité inviter les responsables et citoyens à se projeter dans l'avenir sans attendre la saisine de la Commission nationale du débat public (CNDP) intervenue en décembre 2013, une commission particulière ayant animé le débat de septembre 2014 à janvier 2015³. RFF s'inquiétait en effet du fréquent manque d'anticipation des acteurs publics et privés, confirmé depuis par les chercheurs (Nègre, 2018), et entendait éviter que les acteurs ne se concentrent uniquement sur la liaison entre leur ville et la capitale alors que les enjeux se posent à toutes les échelles (du local au niveau européen) et de façon différenciée selon les territoires (SETEC, 2004). C'est ainsi qu'Elhorst et al. (2004) font varier leur estimation coût-bénéfice du projet ferroviaire Schiphol-Groningue en fonction de trois scénarios européens. Elargir la focale est apparu d'autant plus nécessaire qu'une LGV est indissociable du reste du réseau ferroviaire, lui-même étroitement lié aux autres modes et à la dynamique générale du système de mobilités (Bérion, 1998; Rietveld et Nijkamp, 2000). C'est dans ce contexte que le laboratoire ESO-Rennes a été sollicité pour conduire une prospective de l'accessibilité ferroviaire et du développement territorial de l'Ouest de la France à l'horizon 2040 (Baudelle et al., 2014).

La prospective considère que l'avenir n'est pas écrit d'avance et reste à construire (Fauchard et Mocellin, 2009). Elle vise donc à identifier différents futurs possibles pour les anticiper ou, mieux encore, modeler l'avenir selon ses vœux (Gaudin, 2013). Tout décideur devant être en mesure d'appréhender les enjeux de demain, elle a clairement une fonction stratégique en alertant sur les possibles défis à venir (Durance, 2014). Elle est particulièrement adaptée à la planification spatiale dans la mesure où elle place chacun face aux choix possibles (Courson, 1999; Durance et al., 2007 ; Fourny et Denizot, 2007), ce qui explique son succès précoce et sa diffusion dans le champ de l'aménagement (Vidal-Kratochvil, 2015). Idéalement, elle doit permettre de définir le futur optimal et contribuer à le faire advenir par une stratégie appropriée (Godet et Durance, 2011).

3. [http://cpdp.debatpublic.fr/cpdp-lnobpl/]. 


\section{Une méthodologie prospective adaptée au contexte de recherche}

L'équipe a suivi la méthode par scénarios, éprouvée en prospective territoriale (Loinger, 2006). Cette forme de récit (ou de storytelling) permet de se préparer mentalement à diverses éventualités et d'imaginer le scénario désirable.

L'horizon temporel retenu (2040) devait permettre de prendre en compte les éventuels effets à long terme des investissements ferroviaires en cours et à venir, dont certains ne devaient être lancés qu'après 2030 (figure 1 - planche I).

La construction de scénarios s'effectue en plusieurs étapes (figure 2 - planche II). On a d'abord classiquement (Godet, 2007) recensé l'ensemble des variables internes et externes du système afin d'en identifier les variables-clé, à savoir les éléments exerçant ou susceptibles d'exercer une influence sur le problème étudié (Destatte et Durance, 2009). Pour ce faire, on a listé les changements qu'elles pourraient connaître puis conduit une analyse de type AFOM (Atouts, Faiblesses, Opportunités, Menaces), courante en prospective (Goux-Baudiment, 2006).

L'analyse des stratégies des acteurs vise à déterminer leur degré d'implication face aux enjeux (accord, désaccord, neutralité), leur pouvoir d'influence (de nature politique ou sous forme de lobby) et leur capacité d'action (en termes de compétences et de moyens) selon la méthode dite MACTOR (Matrice des alliances, conflits, tactiques et objectifs) (Godet, 2007). L'examen de leurs positions et de leurs relations permet d'identifier les forces motrices de nature à influencer les variables-clé. L'équipe restreinte missionnée par RFF sur une courte période n'ayant pas les moyens de s'entretenir avec chacun des acteurs ni d'interroger les experts - comme cela est recommandé -, on s'est appuyé sur une étude conduite par un cabinet ayant rencontré les principaux acteurs concernés (MENSIA, 2012) et sur un important rapport issu d'un grand nombre de consultations (CESER, 2012). On a également exploité la presse nationale et régionale qui se fait régulièrement l'écho des prises de position des uns et des autres.

Un atelier de prospective ayant associé des experts extérieurs a permis d'identifier trois enjeux majeurs ${ }^{4}$ :

4. Outre les auteurs, ont contribué Loïc Avry (ESO-Rennes), Bernard Fritsch (ESO-Nantes), Lionel Prigent (université de Bretagne occidentale), Florence Gourlay et Ronan Le Délézir (université de Bretagne Sud). le territoire (en termes d'équilibre, de maillage et de cohésion sociale), la compétitivité (assimilée ici à la recherche de la vitesse et de la performance économique) et l'environnement (comme mobilité et développement durables). La matrice d'évaluation du niveau de sensibilité de chaque acteur à ces enjeux (Baudelle et al., 2014) a été synthétisée (figure 3-planche II).

Les conceptions de chaque groupe ou individu sont bien sûr plus complexes mais l'objectif est d'en donner une représentation accessible afin d'évaluer le rôle qu'ils seraient en mesure de jouer. Certains (dans les angles) s'impliquent clairement dans l'un des trois et font figure de porteurs d'enjeux (stakeholders). D'autres (au centre) s'attachent à plusieurs défis en même temps.

Les éléments à considérer dans l'étude de l'accessibilité ferroviaire et des dynamiques territoriales sont multiples. La méthode permet de sélectionner ceux susceptibles d'influer de manière quasi certaine (Godet et Durance, 2011). On les qualifie de dimensions (ou composantes, sous-systèmes ou questions clés). On est alors en mesure d'élaborer des scénarios partiels (dits sous-scénarios) (tableau 2). De la force motrice commandant chaque sous-scénario résulte une dynamique majeure. Trois ou quatre hypothèses d'évolution sont alors formulées sur la base d'indicateurs appropriés (Baudelle et al., 2014).

\section{Une combinatoire : l'analyse morphologique}

Retenir si peu d'hypothèses pour chacune des variables peut paraître réducteur vu l'infinité de trajectoires imaginables mais l'objectif est précisément d'aider les acteurs à identifier les enjeux majeurs. C'est ici qu'intervient l'analyse dite morphologique (Godet, 2007), phase indispensable de réduction de la complexité par mise en cohérence des sous-scénarios en vue d'aboutir à quelques scénarios globaux. La combinaison théoriquement possible de l'intégralité de nos hypothèses (pourtant délibérément limitées) pour l'ensemble des dimensions aboutit en effet à... 2304 scénarios virtuels. La réduction morphologique permet de s'en tenir à cinq scénarios contrastés (figure 4 - planche III). 


\begin{tabular}{|l|c|c|c|c|}
\hline & Hypothèse A & Hypothèse B & Hypothèse C & Hypothèse D \\
\hline Sous-scénarios & En bonne voie & Tout en douceur & En perte de vitesse & Au rabais \\
\hline Force motrice & Conforme aux plans & Préoccupations écologiques & Freins aux investissements & Libéralisme \\
\hline Dynamique majeure & Développement des projets & Mobilité verte & Sélectivité dans les projets & $\begin{array}{c}\text { Fin des investissements } \\
\text { publics }\end{array}$ \\
\hline
\end{tabular}

I. Sous-scénarios de la dimension « accessibilité »

\begin{tabular}{|l|c|c|c|}
\hline & Hypothèse A & Hypothèse B & Hypothèse C \\
\hline Sous-scénarios & Dans la lignée & Tous les attraits & Perte de vitalité \\
\hline Force motrice & $\begin{array}{c}\text { Dynamisme économique et attracti- } \\
\text { vité hédoniste }\end{array}$ & $\begin{array}{c}\text { Compétitivité et forte attractivité } \\
\text { Dynamique majeure }\end{array}$ & $\begin{array}{c}\text { Perte d’attractivité et moindre } \\
\text { fécondité }\end{array}$ \\
\hline
\end{tabular}

II. Sous-scénarios de la dimension « démographie »

\begin{tabular}{|l|c|c|c|c|}
\hline & Hypothèse A & Hypothèse B & Hypothèse C & Hypothèse D \\
\hline Sous-scénarios & Résistance inégale & Recul productif & Vivre au pays & Spirale de l'expansion \\
\hline Force motrice & Innovation incomplète & Perte de compétitivité & $\begin{array}{c}\text { Développement endogène et } \\
\text { qualitatif }\end{array}$ & $\begin{array}{c}\text { Économie de la connais- } \\
\text { sance réussie }\end{array}$ \\
\hline \begin{tabular}{c} 
Dynamique majeure \\
\hline
\end{tabular} & $\begin{array}{c}\text { Croissance faible et contras- } \\
\text { tée }\end{array}$ & Crise prolongée et récession & Développement équilibré & $\begin{array}{c}\text { Rebonds et cycles de crois- } \\
\text { sance }\end{array}$ \\
\hline
\end{tabular}

III. Sous-scénarios de la dimension « économie »

\begin{tabular}{|l|c|c|c|c|}
\hline & Hypothèse A & Hypothèse B & Hypothèse C & Hypothèse D \\
\hline Sous-scénarios & Équilibre performant & Métropolisation exacerbée & Sans relief & Autonomisation locale \\
\hline Force motrice & $\begin{array}{c}\text { Villes moyennes et coopé- } \\
\text { ration }\end{array}$ & Compétitivité métropolitaine & $\begin{array}{c}\text { Manque d'investissements et } \\
\text { de volontarisme }\end{array}$ & $\begin{array}{c}\text { Stratégies d'attractivité } \\
\text { différenciées }\end{array}$ \\
\hline Dynamique majeure & Polycentrisme intégré & Archipel métropolitain & Nivellement et étiolement & Initiatives locales \\
\hline
\end{tabular}

IV. Sous-scénarios de la dimension « organisation territoriale »

\begin{tabular}{|c|c|c|c|c|}
\hline & Hypothèse A & Hypothèse B & Hypothèse C & Hypothèse D \\
\hline Sous-scénarios & Poursuite de l'individuation & $\begin{array}{l}\text { Chacun connecté à ses } \\
\text { réseaux }\end{array}$ & La réinvention du local & $\begin{array}{l}\text { Le nouvel ordre environne- } \\
\text { mental }\end{array}$ \\
\hline Force motrice & $\begin{array}{c}\text { Individuation des attentes et } \\
\text { des pratiques }\end{array}$ & Nouvelles technologies & Reterritorialisation & $\begin{array}{l}\text { Injonction au développe- } \\
\text { ment durable }\end{array}$ \\
\hline Dynamique majeure & $\begin{array}{l}\text { Affaiblissement de la solida- } \\
\text { rité institutionnelle }\end{array}$ & $\begin{array}{c}\text { Mutation et multiplication } \\
\text { des réseaux }\end{array}$ & $\begin{array}{l}\text { Solidarité de proximité et } \\
\text { modes de vie alternatifs }\end{array}$ & $\begin{array}{l}\text { Impératifs écologiques } \\
\text { globaux }\end{array}$ \\
\hline
\end{tabular}

V. Sous-scénarios de la dimension «société »

\begin{tabular}{|l|c|c|c|}
\hline & Hypothèse A & Hypothèse B & Hypothèse C \\
\hline Sous-scénarios & Dans la norme & Vivre en vert(ueux) & Crise généralisée \\
\hline Force motrice & Respect de normes croissantes & Impératif écologique & Urgence économique \\
\hline Dynamique majeure & $\begin{array}{c}\text { Contraintes croissantes mais aux } \\
\text { effets incertains }\end{array}$ & Développement durable & Primat de l'économique \\
\hline
\end{tabular}

VI. Sous-scénarios de la dimension « environnement »

Tableau 2 : Les hypothèses et les sous-scénarios. En gris, la construction du scénario 1 (cf. figure 4 - planche III) Assumptions and sub-scenarios. In gray, the construction of scenario 1 (see figure 4 - plate III) 


\begin{tabular}{|c|c|c|c|c|c|}
\hline Caractéristiques & $\begin{array}{c}\text { Scénario } 1 \\
\text { L'Ouest sur sa lancée }\end{array}$ & $\begin{array}{c}\text { Scénario } 2 \\
\text { Le déraillement }\end{array}$ & $\begin{array}{c}\text { Scénario } 3 \\
\text { La performance }\end{array}$ & $\begin{array}{c}\text { Scénario } 4 \\
\text { Equilibre et cohésion }\end{array}$ & $\begin{array}{c}\text { Scénario } 5 \\
\text { L'Ouest dans le vert }\end{array}$ \\
\hline Force motrice & $\begin{array}{l}\text { Poursuite des ten- } \\
\text { dances }\end{array}$ & $\begin{array}{c}\text { Perte de vitesse ou crise } \\
\text { généralisée }\end{array}$ & La compétitivité & $\begin{array}{c}\text { Cohésion sociale et } \\
\text { territoriale }\end{array}$ & $\begin{array}{l}\text { La troisième voie: } \\
\text { croissance verte }\end{array}$ \\
\hline Acteurs principaux & $\begin{array}{l}\text { Etat, Région et autres } \\
\text { collectivités territoriales }\end{array}$ & $\begin{array}{l}\text { Capital privé et indi- } \\
\text { vidus }\end{array}$ & $\begin{array}{c}\text { Entreprises et métro- } \\
\text { poles }\end{array}$ & PME et acteurs locaux & $\begin{array}{l}\text { Citoyens et Union } \\
\text { Européenne }\end{array}$ \\
\hline Accessibilité & $\begin{array}{l}\text { Développement des } \\
\text { projets }\end{array}$ & $\begin{array}{c}\text { Sélectivité voire aban- } \\
\text { don des projets faute } \\
\text { d'investissements }\end{array}$ & $\begin{array}{l}\text { Développement des } \\
\text { projets }\end{array}$ & $\begin{array}{l}\text { Développement des } \\
\text { projets }\end{array}$ & Mobilité verte \\
\hline Démographie & Au fil de l'eau & Ralentissement & $\begin{array}{c}\text { Croissance forte et } \\
\text { polarisée }\end{array}$ & Au fil de l'eau & Croissance forte \\
\hline Economie & $\begin{array}{c}\text { Croissance faible et } \\
\text { contrastée }\end{array}$ & $\begin{array}{l}\text { Crise prolongée et } \\
\text { récession }\end{array}$ & $\begin{array}{l}\text { Rebonds et cycles de } \\
\text { croissance }\end{array}$ & $\begin{array}{c}\text { Développement } \\
\text { équilibré }\end{array}$ & $\begin{array}{c}\text { Développement } \\
\text { équilibré }\end{array}$ \\
\hline $\begin{array}{l}\text { Organisation terri- } \\
\text { toriale }\end{array}$ & Polycentrisme intégré & $\begin{array}{c}\text { Nivellement et étiole- } \\
\text { ment }\end{array}$ & Archipel métropolitain & Initiatives locales & Polycentrisme intégré \\
\hline Société & $\begin{array}{l}\text { Affaiblissement de la } \\
\text { solidarité institution- } \\
\text { nelle }\end{array}$ & $\begin{array}{l}\text { Chacun pour soi ou } \\
\text { repli communautaire }\end{array}$ & $\begin{array}{l}\text { Mutation et multiplica- } \\
\text { tion des réseaux }\end{array}$ & $\begin{array}{l}\text { Solidarité de proxi- } \\
\text { mité et modes de vie } \\
\text { alternatifs }\end{array}$ & $\begin{array}{c}\text { Impératifs écologiques } \\
\text { globaux }\end{array}$ \\
\hline Environnement & $\begin{array}{c}\text { Contraintes crois- } \\
\text { santes mais aux effets } \\
\text { incertains }\end{array}$ & Primat de l'économique & $\begin{array}{c}\text { Contraintes crois- } \\
\text { santes mais aux effets } \\
\text { incertains }\end{array}$ & Développement durable & Développement durable \\
\hline
\end{tabular}

Tableau 3 : Résumé des cinq scénarios Summary of the five scenarios

\section{LES SCÉNARIOS POUR 2040 ET LEURS IMPLICATIONS POUR L'AMÉNAGEMENT}

\section{Cinq scénarios pour l'Ouest ferroviaire}

Ces scénarios sont les suivants (figure 4 - planche III et tableau 3) :

L'Ouest sur sa lancée poursuit les tendances récentes

Le déraillement entrevoit un Ouest en perte de vitesse voire en crise généralisée

La performance est guidé par la compétitivité

Équilibre et cohésion est orienté vers la cohésion sociale et territoriale

L'Ouest dans le vert explore une troisième voie : la croissance verte

Ces scénarios finaux ont été positionnés par rapport aux trois principaux défis identifiés (figure 5- planche III).

\section{Scénario 1 : L'Ouest sur sa lancée, scénario tendanciel (figure 6 - planche IV)}

Ce scénario prolonge les dynamiques à l'œuvre depuis une trentaine d'années : les projets ferroviaires se poursuivent (mais sans certitude d'aboutissement). La croissance économique et démographique ralentit et demeure contrastée, creusant les écarts entre territoires. En 2040, Nantes et Rennes, portées par l'économie de la connaissance vectrice de métropolisation, surclassent les autres villes. Un scénario tendanciel ne signifie pas que l'avenir ressemblera au présent. Une seconde erreur consiste à confondre scénario tendanciel et évolution spontanée, comme si le scénario « au fil de l'eau » coulait de source, alors qu'aucune évolution ne s'impose d'elle-même : il n'y a ni fatalité du retard, ni assurance de poursuite du développement.

\section{Scénario 2 : Le déraillement, scénario catastrophe (figure 7 - planche V)}

Ce scénario repoussoir voit l'Ouest décliner faute d'avoir modernisé ses infrastructures suite à une crise économique mondiale qui conduit à l'abandon des grands projets. L'aérien plus libéralisé plombe un ferroviaire surendetté que les collectivités ne peuvent plus soutenir, entraînant réduction drastique des dessertes, fermeture de gares et de lignes. L'Ouest redevient une périphérie où les villes en difficulté se font concurrence sans enrayer la polarisation francilienne. Seules Nantes (plus puissante) et Rennes (plus proche) tirent parti de l'orbite parisienne. 
Scénario 3 : La performance, scénario de la compétitivité (figure 8 planche VI)

Ce scénario est à l'opposé : le souci de la compétitivité guide le devenir régional. Le succès est soutenu par la réalisation d'infrastructures performantes (LGV, aéroports agrandis, ports modernisés, nouveaux systèmes de télécommunication). Dans un premier temps se produit une métropolisation exacerbée au profit de Rennes et Nantes mais dans une seconde phase, grâce à un maillage serré de réseaux, la croissance se diffuse aux autres grandes villes et aux villes moyennes offrant aménités résidentielles et moindres coûts.

\section{Scénario 4 : Équilibre et cohésion, scénario territorial (figure 9 - planche VII)}

Ce scénario donne la priorité à la cohésion sociale et territoriale, c'est-à-dire à la limitation des inégalités. Sa dynamique est endogène : subsidiarité, retour au local, solidarités de proximité. Entrepreneurs locaux, élus et associations s'organisent pour assurer un réseau dense de transports. Intercommunalité et péréquation fiscale régionale tentent d'assurer l'équirépartition du développement sans y parvenir car l'Ouest est éclaté en une mosaïque de bassins hétérogènes. Comme l'insertion dans des réseaux élargis est indispensable au succès d'un modèle enraciné, la prééminence de Nantes comme gateway city internationale est acceptée. Le modèle est proche de l'Allemagne du Sud, avec un encastrement territorial d'acteurs fidèles à leur région mais tournés vers l'innovation.

\section{Scénario 5 : L'Ouest dans le vert, scénario du développement durable (figure 10 - planche VIII)}

L'Ouest s'inspire de la voie alternative d'une croissance écologique sur le modèle nordique par un cheminement en douceur ou suite à une catastrophe majeure (marée noire, épizootie, accident nucléaire, ouragan...) qui provoque un choc. Le transport aérien subit un arrêt considérable. Les LGV sont contestées du fait de leur impact écologique mais le rail classique modernisé connaît un nouvel essor grâce à l'intermodalité douce. Les progrès de l'e-ccessibilité optimisant l'accessibilité numérique réduisent la mobilité physique. La croissance est géographiquement équilibrée.

\section{Les défis pour l'aménagement du territoire}

La prospective prépare la stratégie, elle comporte donc une dimension politique et praxéologique (Gonod, 2005). À chacun de ces scénarios est associé un défi majeur pour l'action aménagiste (tableau 4).

Dans le scénario tendanciel (1), la réalisation des seuls projets ferroviaires actuels pourrait se révéler insuffisante au regard des exigences d'accessibilité future, conduisant à un décrochage par rapport à d'autres parties du pays. Dans le déraillement, le défi majeur est de se montrer proactif en investissant malgré la crise dans les transports et l'innovation, indispensables à la compétitivité et à l'attractivité. La cohésion territoriale constitue l'enjeu majeur du scénario de la performance où le passage à une économie de la connaissance centrée sur les métropoles ne saurait exclure les espaces hors métropoles, ce qui suppose de compléter les équipements d'envergure par un maillage dense associant les villes moyennes. L'enjeu du scénario 4 est de concilier autonomie locale et ouverture internationale, indispensable à la réussite d'un modèle de développement enraciné. Enfin, le principal défi du scénario 5 est d'assurer la rentabilité de la transition risquée vers une économie verte qui impose des investissements considérables et risqués dans des systèmes de communication soutenables.

\begin{tabular}{|l|c|c|c|c|c|}
\hline Scénarios & $\begin{array}{c}\text { 1. L'Ouest sur sa } \\
\text { lancée }\end{array}$ & $\begin{array}{c}\text { 2. Le déraille- } \\
\text { ment }\end{array}$ & $\begin{array}{c}\text { 3. La perfor- } \\
\text { mance }\end{array}$ & $\begin{array}{c}\text { 4. Équilibre et } \\
\text { cohésion }\end{array}$ & $\begin{array}{c}\text { 5. L'Ouest dans } \\
\text { le vert }\end{array}$ \\
\hline $\begin{array}{l}\text { Défis pour l'amé- } \\
\text { nagement du } \\
\text { territoire }\end{array}$ & $\begin{array}{c}\text { Mener les projets } \\
\text { jusqu'à leur terme } \\
\text { équitablement }\end{array}$ & $\begin{array}{c}\text { Faire face à la } \\
\text { récession }\end{array}$ & $\begin{array}{c}\text { Garantir la cohé- } \\
\text { sion territoriale }\end{array}$ & $\begin{array}{c}\text { Veiller à l'insertion } \\
\text { internationale }\end{array}$ & $\begin{array}{c}\text { Réaliser la crois- } \\
\text { sance par l'écologie }\end{array}$ \\
\hline
\end{tabular}

Tableau 4 : Cinq scénarios et autant de défis pour l'aménagement Five scenarios: all challenges for planning 
Chacun reconnaît la nécessité de relever ces impératifs de long terme mais des difficultés surgissent dès qu'il s'agit d'arbitrer entre ces priorités, soumettant les décideurs à des injonctions contradictoires, sachant que toutes les valeurs ne sont pas toujours conciliables en matière d'aménagement. Les scénarios esquissent cinq réponses possibles. À chacun de se positionner, de refuser ou de défendre telles options, mais aussi d'en imaginer d'autres. C'est la vocation de la prospective que d'aider les acteurs à forger l'image du futur souhaitable. D'où le choix d'un nombre délibérément restreint de scénarios contrastés, sans ambition de réaliser à ce stade une simulation géoprospective précise à partir d'indicateurs fins (Fusco, 2012). C'est pourquoi on n'a pas non plus recommandé de scénario normatif (ou souhaitable), considérant que c'était aux décideurs (élus, investisseurs) et aux citoyens de définir leurs priorités.

\section{Conclusion}

Les scénarios donnent à voir quelques trajectoires possibles. Classiquement, le scénario tendanciel poursuit les processus en cours. Ce n'est pas nécessairement le plus probable mais il permet de représenter l'avenir en l'absence de rupture majeure. Le scénario catastrophe alerte délibérément sur les risques encourus en cas de renoncement complet ou partiel aux projets de modernisation ferroviaire. Ce n'est pas le plus incertain. Les trois scénarios alternatifs explorent d'autres trajectoires en fonction de forces motrices distinctes.

Quelle voie empruntera l'Ouest? Nous n'en savons rien, ne serait-ce qu'en raison des limites inhérentes à la prospective régionale par scénarios (Loinger, 2004) qui n'est du reste qu'une des techniques possibles (Fahri et al., 2002). Ces scénarios esquissent seulement cinq futurs envisageables cohérents et réalistes qui sont autant d'éventualités soumises aux parties prenantes. Aux décideurs ensuite d'agir pour que se réalise l'avenir le plus désirable à leurs yeux. Cela suppose des arbitrages. Les récents rapports Duron (2018) et Spinetta (2018) esquissent des pistes de réforme partiellement convergentes de la carte et de l'organisation ferroviaires nationales : priorité aux mobilités du quotidien, à la modernisation des infrastructures et aux dessertes à grande vitesse entre métropoles ${ }^{5}$. Cela ne ferme pas pour autant l'éventail des trajectoires futures, une erreur souvent commise en prospective étant de se laisser influencer par les tendances récentes voire par l'actualité immédiate.

\section{Bibliographie}

Agences d’urbanisme de Bretagne, 2010. Les territoires bretons et le projet Bretagne à Grande Vitesse, Brest, ADEUPa, 43 p. [en ligne], consulté le 30 avril 2017. [https://www. adeupa-brest.fr/system/files/publications/les_territoires_ bretons_et_le_projet_bgv.pdf].

Alabalate D., Bel G., 2010. High-speed rail: lessons for policy makers from experiences abroad, Barcelone, IREA Working Papers, 34 p. [http://www.ub.edu/irea/working_ papers/2010/201003.pdf].

Ahlfeldt M., Feddersen A., 2015. From periphery to core: measuring agglomeration effects using high-speed rail, SERC Discussion Paper, $\mathrm{n}^{\circ}$ 172, London School of Economics, 63 p. [http://www.spatialeconomics.ac.uk/textonly/ SERC/publications/download/sercdp0172.pdf].

АмтRAк, 2012. A vision for high-speed rail in the North-East Corridor, New York, 42 p. [https://www.amtrak.com/ ccurl/453/325/Amtrak-Vision-for-the-Northeast-Corridor. $\mathrm{pdf}]$.

AUDIAR (Agence d'urbanisme et de développement intercommunal de l'agglomération rennaise), 2016. EuroRennes et l'attractivité rennaise post-2020. Le point de vue des PME locales, Rennes, Contribution prospective, no 5, 16 p. [en ligne], consulté le 29 avril 2017. [http://www.audiar.org/ sites/default/files/documents/etudes/contrib5_eurorennes_ post2020_web.pdf].

Auphan E., 1975. Les nœuds ferroviaires, phénomène résiduel ou points forts de l'espace régional, L'Espace Géographique, no 2, p. 127-129.

Banister D., Berechman J., 2001. Transport investment and the promotion of economic growth, Journal of Transport Geography, no 9, p. 209-218.

Baudelle G., 2016. La grande vitesse ferroviaire accélère-t-elle la croissance des villes?, Place Publique (Rennes), n 39, p. $40-44$.

Baudelle G., Brugioni I., Lepetit A., 2014. L'Ouest et le rail. Enjeux et prospective, Rennes, Presses universitaires de Rennes, 159 p.

Bazin S., Beckerich C., Delaplace M., 2006. La LGV Est européenne en Champagne-Ardenne : quels effets sur la cohésion territoriale champardennaise?, Revue d'Économie Régionale et Urbaine, $\mathrm{n}^{\circ}$ 2, p. 245-261.

Bazin S., Beckerich C., Delaplace M., 2010. Desserte ferroviaire à grande vitesse, activation des ressources spécifiques

5. La Loi « Pour un nouveau pacte ferroviaire » adoptée en juillet 2018 a suivi plusieurs recommandations du rapport Spinetta. Pour sa part, l'ouverture progressive à la concurrence des marchés nationaux des voyageurs résulte du « $4^{\mathrm{e}}$ paquet ferroviaire » adopté par le Parlement européen en décembre 2016. 
et développement du tourisme : le cas de l'agglomération rémoise, Belgeo [Online], 1-2 | 2010. DOI : 10.4000/belgeo.6669.

Bazin S., Beckerich C., Blanquart C., Delaplace M., VanDEnbossche L., 2011. Grande vitesse ferroviaire et développement économique local : une revue de la littérature, Recherche, Transports et Sécurité, vol. 27, no 106, p. 215-238.

Bazin S., Beckerich C., Delaplace M., 2013. Desserte TGV et villes petites et moyennes. Une illustration par le cas du tourisme à Arras, Auray, Charleville-Mézières et Saverne, Les Cahiers Scientifiques du Transport, nº 63, p. 33-62.

Bazin S., Beckerich C., Delaplace M., Masson S., Petiot R., 2004. La LGV : un outil d'ouverture des espaces et de renforcement de l'attractivité touristique, Revue de l'Economie Méridionale, vol. 52, $\mathrm{n}^{\circ}$ 1-2, p. 57-83.

Bél i Queralt G., 2010. España, capital París. Origen y apoteosis del estado radial: del Madrid sede cortesana a la «capital total», Barcelone, Destino, 325 p.

Bellet C., Alonso P., Casellas A., 2010. Infrastructuras de transporte y territorio. Los efectos estructurantes de la llegada del tren de alta velocidad en España, Boletín de la Asociación de Geógrafos Españoles, n 52, p. 143-163.

Bellet C., Alonso P., Gutiérrez A., 2012. The High-Speed rail in Spanish cities: urban integration and local strategies for socio economic development, in UREÑA J.M. (dir.), Territorial implications of High-Speed rail: a Spanish perspective, Aldershot, Ashgate, p. 163-196.

Bellet Sanfeliu C., Santos Ganges L. 2016. The high-speed rail project as an urban redevelopment tool, Belgeo [En ligne], 3-2016, consulté le 15 mai 2017. DOI: 10.4000/ belgeo. 18153 .

Berechman J., 2003. Transportation. Economic aspects of Roman highway development: the case of Via Appia, Transportation Research Part A, no 37, p. 453-478. DOI: 10.1016/ S0965-8564(02)00056-3.

BERECHMAN J., 2009. The evaluation of transportation investment projects, New York/Oxon, Routledge, 404 p.

BÉRion P., 1998. Analyser les mobilités et le rayonnement des villes pour révéler les effets territoriaux des grandes infrastructures de transport, Cahiers Scientifiques $d u$ Transport, $n^{0} 33$, p. 109-127.

Berroir S., Cattan N., Guérois M., Paulus F., VaccianiMarcuzzo C., 2012. Les systèmes urbains français. Synthèse, Travaux en ligne, no 10, DATAR. [http://www.avecnet. net/wp-content/doc/Documentation/FR/Les-Systemesurbains-francais-Synthese-de-letude-de-Geographie-cites. pdf].

Bertrand L., Billard G., 2018. Introduction, Norois, n² 248, p. $1-7$.

Bretagnolle A., 1999. Les systèmes de villes dans l'espacetemps : effets de l'accroissement de la vitesse des déplacements sur la taille et l'espacement des villes, Thèse de doctorat de Géographie, Université Paris 1, 280 p.

Bretagnolle A., 2003. Vitesse des transports et sélection hiérarchique entre les villes françaises, in Pumain D., Mattei M.-F. (dir.), Données urbaines 4, Paris, Anthropos, p. 309-323.
Bretagnolle A., 2009. Villes et réseaux de transport: des interactions dans la longue durée (France, Europe, États-Unis), Habilitation à Diriger des Recherches, Université Paris 1, [https://tel.archives-ouvertes.fr/tel-00459720/document].

Bretagnolle A., Pumain D., Rozenblat C., 1998. Space-time contraction and the dynamics of urban systems, Cybergeo, $\mathrm{n}^{\circ} 61,12 \mathrm{p}$.

Brotchie J., 1991. Fast rail networks and socio economic impacts, in Brotchie J., Batty P. Hall P., Newton J. (dir.), Cities of the $21^{\text {st }}$ Century: new technologies and spatial systems, New York, Longman, p. 25-37.

Button K, 2017. High-Speed railways: Do they produce economic growth?, Arlington, Mercatus Center, George Madison University, 32 p. [https:/www.mercatus.org/system/files/ mercatus-button-high-speed-railways-vl.pdf].

CEMT (Conférence européenne des ministres des transports), 2001. Évaluer les avantages des transports, Paris, Editions de l'OCDE, $227 \mathrm{p}$.

CEMT, 2002. Transport et développement économique. $119^{e}$ table ronde d'économie des transports, Paris, OCDE, 209 p.

CESER Bretagne, 2012. Optimiser les projets Bretagne à Grande Vitesse, Dupont J.-P., Ollivard P. (rapp.), Rennes, Conseil économique, social et environnemental, $390 \mathrm{p}$.

Chapulut J.-N. (rapp.), 2001. Bilan LOTI du TGV Atlantique, Rapport du Conseil Général des Ponts et Chaussées, Paris-La Défense, 46 p. [http://www.cgedd.developpementdurable.gouv.fr/IMG/pdf/TGV_Atl_cle2668c5.pdf].

Chen G., Abreu e Silva J. De, 2013. The regional impacts of high speed rail. A review of methods and models, Transportation Letters, vol. 5, $\mathrm{n}^{\circ} 3$, p. 131-143.

Chen G., Abreu e Silva J. De, 2014. Estimating the provincial economic impacts of High-speed rail in Spain: an application of structural equation modelling, Procedia-Social and Behavioral Sciences, vol. 111, p. 157-165. doi.org/10.1016/j. sbspro.2014.01.048.

Cheng Y.-S., Lo B.P., Vickerman R., 2013. High-speed rail networks and economic integration in China and Europe, $13^{\text {th }}$ World Conference on Transport Research Selected Proceedings, Rio de Janeiro, 24 p. [http://www.wctrs-society. com/wp/wp-content/uploads/abstracts/rio/selected/2368. pdf].

Chevalier J. (coord.), 1995-1997. Le Mans, 6 ans après l'arrivée du TGV, Rapport à la DATAR et à l'Observatoire économique et statistique des transports, ESO, Université du Maine, Le Mans, 2 vol., 70 et 43 p.

Courson J. De, 1999. La prospective des territoires. Concepts, méthodes, résultats, Lyon, Éditions du CERTU, 124 p.

Crozet Y., 2014. Accessibilité des transports terrestres et développement économique : nous devons changer d'époque!, Annales des Mines - Responsabilité et environnement, vol. 3, no 75, p. 30-34. DOI : 10.3917/re.075.0030.

Crozet Y., 2015. High speed rail and urban dynamics: wider or targeted economic effects?, Colloque High-speed rail and the city: urban dynamics and tourism, Champs-sur-Marne, 21-23 Janvier, Université Paris-Est. 
DATAR, 1994. Dossier Prospective et territoires, Paris, La Documentation Française, 163 p.

Delaplace M., Bazin S., Beckerich C., Blanquart C., 2013. Les enjeux et opportunités de la Grande Vitesse Ferroviaire en termes de développement local et de développement durable: une analyse fondée sur la production et l'appropriation des innovations de services, Université de Reims-ChampagneArdenne. [https://halshs.archives-ouvertes.fr/hal-01098691/ document, 26/01/2016].

Delaplace M., Dobruszkes F., 2013. Analysing the relationship between high-speed rail and territories: a scientific field still in-progress, Recherche Transports et Sécurité, $n^{\circ} 29$, p. 155-160.

Delaplace M., Dobruszkes F., 2016. Editorial: thinking beyond the cost-benefit analysis: the wider impact of highspeed rail on local development, Belgeo [En ligne], 3-2016, consulté le 14 mai 2017. [http://belgeo.revues.org/18166].

Destattte P., Durance P. (dir.), 2009. Les mots-clés de la prospective territoriale, Paris, La Documentation française, $64 \mathrm{p}$.

Dornbusch J., 1997. Nantes, sept ans après l'arrivée du TGV Atlantique, Notes de synthèse du SES, mai-juin, 4 p. [http:// temis.documentation.developpement-durable.gouv.fr/pj/ NS/NS_111_1.pdf].

Durance P. (dir.), 2014. La prospective stratégique en action, Paris, Odile Jacob, 400 p.

Durance P. et al., 2007. La prospective territoriale : pour quoi faire? Comment faire?, Cahiers du LIPSOR, Série Recherche, $\mathrm{n}^{\circ}$ 7, $142 \mathrm{p}$.

Duranton G., Turner M.A., 2012. Urban growth and transportation, Review of Economic Studies, vol. 79, $\mathrm{n}^{\circ} 4$, p. 1407-1440.

Duron P. (prés.), 2018. Mobilités du quotidien : répondre aux urgences et préparer l'avenir, Paris, Conseil d'orientation des infrastructures, rapport à Madame la Ministre, 207 p. [https:/www.ecologique-solidaire.gouv.fr/sites/default/ files/2018.02.01_rapport_coi.pdf].

Elhorst J.P., Oosterhaven J.R., 2004. Integral cost-benefit analysis of Maglev technology under market imperfection, SOM Research Report, Université de Groningue, 36 p. [http:// www.rug.nl/research/portal/files/3089364/04c22.pdf].

Esteban M., 1998. La alta velocidad ferroviaria en la Union Europea. Su impacto urbano en Francia y España, Geographícalia, $n^{\circ} 36$, p. 19-32.

Facchinetti-Mannone V., 2009. Location of high speed rail stations in French medium-size city and their mobility and territorial implications: central, peripheral and bis (both central and peripheral in the same city), International Conference City Futures 09, Madrid, 4-6 juin, 17 p.

Facchinetti-Mannone V., Bellet C., Ribalaygua C., Richer C., 2013. Les petites agglomérations françaises et espagnoles face à la grande vitesse ferroviaire : comment convertir l'accessibilité en attractivité?, Les Cahiers Scientifiques $d u$ Transport, $n^{\circ} 63$, p. 3-31.

FAHri F. et al., 2002. Guide pratique de prospective territoriale en France, Bruxelles, Commission européenne, DG Recherche, Unité Prospective scientifique et technologique, $149 \mathrm{p}$.
Fauchard L., Mocellin P., 2009. Conduire une démarche de prospective territoriale, Paris, L'Harmattan, 146 p.

Fourny M.-C., Denizot D., 2007. La prospective territoriale, révélateur et outil d'une action publique territorialisée, in Dodier R., Rouyer A., Séchet R. (dir.), Territoires en action et dans l'action, Rennes, Presses universitaires de Rennes, p. 29-44.

Fujita M., Krugman P., Venables A.J., 1999. The spatial economy. Cities, regions, and international trade, Cambridge (Mass.), M.I.T. Press, 382 p.

Fusco G., 2012. Démarche géo-prospective et modélisation causale probabiliste, Cybergeo : European Journal of Geography [En ligne], mis en ligne le 20 juillet 2012, consulté le 6 mars 2018. [http://journals.openedition.org/cybergeo/25423; DOI : 10.4000/cybergeo.25423].

Garmendia M., Ribalaygua C., Ureña J. M., 2012a. High speed rail: implication for cities, Cities, vol. 29, nº 2 , p. 26-31.

Garmendia M., Romero V., Ureña J. M., Coronado J. M., Vickerman R., 2012b. High-speed rail opportunities around metropolitan regions: the cases of Madrid and London, Journal of Infrastructure Systems, vol. 18, no 4, p. 305-313.

Gaudin T., 2013. La prospective, Paris, PUF, coll. «Que saisje? ", 128 p.

Godet M., 2007 (3e éd.). Manuel de prospective stratégique, L'art et la méthode, T. 2, Paris, Dunod, 448 p. [http://www. laprospective.fr/dyn/francais/ouvrages/la_prospective_strategique/t 1-manuel-de-prospective-strategique-dunod-2007. pdf] et [http://www.laprospective.fr/dyn/francais/ouvrages/ la_prospective_strategique/t2--manuel-de-prospective-strategique-dunod-2007.pdf].

Godet M., Durance P., 2011 (2e éd.). La prospective stratégique. Pour les entreprises et les territoires, Paris, Dunod, 224 p.

Gonod P., 2005. L'hypothèse générale de la prospective anthropolitique, Colloque «Intelligence de la complexité », 23-30 juin, Cerisy-la-Salle, 47 p. [http://archive.mcxapc.org/docs/ ateliers/0305gonod 1.pdf].

Goux-Baudiment F., 2006. Donner du futur aux territoires. Guide de prospective territoriale à l'usage des acteurs locaux, Lyon, CERTU, coll. «Collections du CERTU », 276 p.

Gutiérrez J., 2001. Location, economic potential and daily accessibility: an analysis of the accessibility impact of the high-speed line Madrid-Barcelona-French border, Journal of Transport Geography, vol. 9, nº 4, p. 229-242.

Gutiérrez Puebla J., 2004. The high-speed train and its spatial effects, Investigationes Regionales, n 5, p. 199-221.

Gutiérrez J., Condeço-Melhorado A., Martín J.C., 2010. Using accessibility indicators and GIS to assess spatial spillovers of transport infrastructure investment, Journal of Transport Geography, vol. 18, n 1, p. 141-152.

Hall P., 2009. Magic carpets and seamless webs: opportunities and constraints for high-speed trains in Europe, Built Environment, vol. 35, $\mathrm{n}^{\circ}$ 1, p. 59-69.

Huart Y., 1994. Les effets des TGV sur l'aménagement des territoires, OEST Synthèse, Septembre, 4 p. [http://temis. documentation.developpement-durable.gouv.fr/pj/NS/ NS_083_6.pdf]. 
Jensen-Butler C., Madsen B., 2005. Transport and regional growth, in Button K.J., Hensher D.A. (dir.), Handbook of transport strategy, policy and institutions, Oxford, Elsevier, vol. 6, p. 191-223.

KLEIN O., 1998. Le TGV-Atlantique et les évolutions de la mobilité : entre crise et concurrence, Les Cahiers Scientifiques $d u$ Transport, p. 57-83.

LEVInson D.M., 2012. Accessibility impacts of high-speed rail, Journal of Transport Geography, n 22, p. 288-291.

Loinger G. (dir.), 2004. La Prospective régionale, de chemin en desseins, La Tour-d'Aigues, Éditions de L'Aube, 275 p.

Loinger G., 2006. Développement des territoires et prospective stratégique, Paris, L'Harmattan, 230 p.

Loukaitou-Sideris A., Higgins H., Piven M., Wei W., 2013. Tracks to change or mixed signals? A review of the AngloSaxon literature on the economic and spatial impacts of High-Speed rail, Transport Reviews, p. 617-633. [http:// dx.doi.org/10.1080/01441647.2013.836578].

Meer A., Ribalaygua C., Martín E., 2012. High-Speed rail and regional accessibility, in UREÑA J.M. (dir.), Territorial implications of High-Speed rail: a Spanish perspective, Aldershot, Ashgate, p. 197-216.

Ménerault P., Barré A. (dir.), 2001. Gares et quartiers de gares: signes et marges. Lille, Rennes et expériences internationales (Italie, Japon, Pays-Bas), Actes du Séminaire international, Villeneuve-d'Ascq, 22 mars 1999, 216 p.

MENSIA, 2012. Dossier de saisine de la Commission Nationale du Débat Public. Annexe : dossier de contexte, Paris, MENSIA Conseil/RFF, novembre 2012, 23 p.

Mignerey P., 2012. Les effets territoriaux de la grande vitesse ferroviaire en France, Paris, La Documentation française, DATAR, 94 p.

Mohino I., Loukaitou-Sideris A., Ureña J. M., 2014. Impacts of high-speed rail on metropolitan integration: an examination of London, Madrid and Paris, International Planning Studies, vol. 19, no 3-4, p. 306-334. [http://dx.doi.org/10.1 080/13563475.2014.950638].

NÈGRE R., 2018. Regards prospectifs spatialisés des entreprises bretonnes et ligériennes sur l'accessibilité ferroviaire nouvelle, Norois [En ligne], no 248.

Nègre R., Baudelle G., 2016. City planning related to the completion of the new High Speed Line in Rennes, Brittany. The unexpected competition between central projects and peripheral private investments, Belgeo [Online], 3 | 2016. [http://belgeo.revues.org/18122].

OCDE, 2008. Bénéfices économiques élargis du secteur des transports. Instruments d'investissement et d'évaluation macro-, méso- et micro-économiques, Forum international sur les transports, 140, Paris, Editions de l'OCDE, 222 p.

Offner J.-M., 1993. Les effets structurants du transport: mythe politique, mystification scientifique, L'espace Géographique, $\mathrm{n}^{\circ} 3$, p. 233-242.

Offner J.-M., 2009. En finir avec les effets structurants!, Traits Urbains, décembre, p. 9.

Offner J.-M. et al., 2014. Les effets structurants des infrastructures de transport, L'Espace Géographique, n 1, p. 51-67. DOI : 10.3917/eg.431.0051.
Oosterhaven J., KnaAp T., 2003. Spatial economic impacts of transport infrastructure investments, in Pearman A., MaCKIE P., NellthorP J. (dir.), Transport projects, programmes and policies: evaluation needs and capabilities, Ashgate, Aldershot, p. 87-101.

Plassard F., 2003. Transport et territoire, Paris, La Documentation française, $97 \mathrm{p}$.

Puga D., 2002. European regional policies in light of recent location theories, Journal of Economic Geography, $\mathrm{n}^{\circ} 2$, p. 373-406.

Pumain D., 1982. Chemin de fer et croissance urbaine en France au XIX ${ }^{\mathrm{e}}$ siècle, Annales de Géographie, vol. 91, nº 507, p. $529-550$.

Quéré T., 2013. Les effets du TGV sur l'aménagement du territoire, Colloque $150^{e}$ anniversaire de l'arrivée $d u$ train à Saint-Brieuc, Saint-Brieuc, 12 octobre.

Richer C. (dir.), 2014. Évaluation des projets ferroviaires à grande vitesse. Aspects liés à l'accessibilité aux petites villes et territoires intermédiaires, réflexions issues d'études de cas en Europe, rapport au Cerema, Paris, IFFSTAR-DEST, 74 p. [http://www.infra-transports-materiaux.cerema.fr/IMG/ pdf/1431w-rapport_tgv_.pdf].

Rietveld P., Bruinsma F., 1998. Is transport infrastructure effective?, Berlin, Springer, 365 p.

Rietveld P., Nijkamp P., 2000. Transport infrastructure and regional development, in Polak J. B., Heertje A. (dir.), Analytical transport economics. An international perspective, Cheltenham, Edward Elgar, p. 208-234.

Rozenblat C., 2004. Tissus de villes. Réseaux et systèmes urbains en Europe, Habilitation à diriger des recherches, Université de Montpellier III, 193 p. [https://doc.rero.ch/record/8547/ files/HDR_C.Rozenblat.pdf].

Rus J. DE (dir.), 2012 (2 éd.). Economic analysis of high speed rail in Europe, Bilbao, Fundación BBVA, 132 p. [http:// www.fbbva.es/TLFU/dat/inf_web_economic_analysis.pdf].

Rus J. DE, NASH C.A., 2007. In what circumstances is investment in HSR worthwhile?, MPRA Paper, $\mathrm{n}^{\circ}$ 8044, Institute for Transport Studies, University of Leeds, 36 p. [https:// mpra.ub.uni-muenchen.de/8044/1/MPRA_paper_8044. pdf].

Saladié Ò., Clavé S. A., Gutiérrez A., 2016. Measuring the influence of the Camp de Tarragona high-speed rail station on first-time and repeat tourists visiting a coastal destination, Belgeo [En ligne], 3-2016, consulté le 15 mai 2017. DOI : 10.4000/belgeo. 17889.

SALmon H., 2013. Les effets attendus de la ligne à grande vitesse Bretagne-Pays de la Loire sur les principales villes desservies: le point de vue des acteurs du développement économique et du transport, Master Aménagement et Collectivités Territoriales, Université Rennes 2, 129 p. [en ligne], consulté le 30 avril 2017. [http:/www.ere-lgv-bpl.com/home/observatoire/publications/mainContent/liste-dactualites/les-effetsattendus-de-la-ligne.html].

Sasaki K., Ohashi T., Ando A., 1997. High-speed rail transit impact on regional systems: does the Shinkansen contribute to dispersion?, The Annals of Regional Science, $\mathrm{n}^{\circ} 31$, p. 77-98. 
SETEC, 2004. Analyse prospective des effets potentiels de la LGV PACA, Paris/Nice, SETEC \& UMR Espaces, Université Nice Sophia Antipolis, 57 p. [http://cpdp.debatpublic.fr/ cpdp-lgvpaca/docs/pdf/etudes/etude_relative/LGV-PACAEffetTerritoire-Volet4-juin04.PDF].

SETEC, 2010. Effets territoriaux de la grande vitesse ferroviaire. Ligne à Grande Vitesse Paris-Orléans-Clermont-FerrandLyon, Paris, 32 p. [http://www.cg58.fr/IMG/pdf/Effets-territoriaux-de-la-grande-vitesse-ferroviaire.pdf].

Spinetta J.-C. (rapp.), 2018. L'avenir du Transport ferroviaire, rapport au $1^{\text {er }}$ Ministre, Paris, 127 p. [https://www.ecologique-solidaire.gouv.fr/sites/default/files/2018.02.15_Rapport-Avenir-du-transport-ferroviaire.pdf].

Stein N.E.G., 2013. Spatial dimensions of high-speed rail: intermediate cities, inter-jurisdictional planning, and the implications for high-speed rail in Portugal, Master of Science in Transportation, Master in City Planning, Cambridge (Mass.), Massachusetts Institute of Technology, 204 p. [http://web.mit.edu/hsr-group/documents/Stein_Thesis_2013.pdf].

Terrin J.-J. (dir.), 2011. Gares et dynamiques urbaines. Les enjeux de la grande vitesse, Marseille, Parenthèses, 223 p.

Ureña J. M., Ménerault P., Garmendia M., 2009. The highspeed rail challenge for big intermediate cities: a national, regional and local perspective, Cities, vol. 26, n 5, p. 266-279.

Van den Berg L., Pol P., 1998. The European high-speed train and urban development. Experiences in fourteen European urban regions, Aldershot, Ashgate, 264 p.
Vickerman R., 1997. High-speed rail in Europe: experience and issues for future development, The Annals of Regional Science, no 31 , p. 21-38.

Vickerman R., 2006. Indirect and wider economic impacts of high-speed rail, 4th conference on railroad industry structure, competition and investment, Octobre, Madrid, 18 p.

Vickerman R., 2014. High-speed rail and regional development: the case of intermediate stations, Journal of Transport Geography, no 42, p. 157-165. DOI: 10.1016/j.jtrangeo.2014.06.008.

Vickerman R., 2017. Wider impacts of megaprojects. Curse or cure?, in Flyvbjerg B. (dir.), The Oxford handbook of megaproject management, Oxford, Oxford University Press, p. 389-405.

Vickerman R., Spiekermann K., Wegener M., 1999, Accessibility and regional development in Europe, Regional Studies, no 33, p. 1-15.

Vidal-KratochVil C., 2015. La prospective territoriale dans tous ses états. Rationalités, savoirs et pratiques de la prospective (1957-2014), Thèse de Géographie, Lyon, École Normale Supérieure.

Wang J., Charles M.B., 2010. The potential impacts of high speed rail on regional economic development in Australia: towards a multi-regional input-output approach, $7^{\text {th }}$ world congress on high speed rail, Pékin, 7-9 Décembre, p. 1-17. [http://epubs.scu.edu.au/cgi/viewcontent. coirarticle $=1097 \&$ context $=$ bus_pubs]. 